دراسة الطباقية الحياتية للفورامنيفرا الطافية لتكوين شرانش وطبيعة تماسه مع تكوين عليجي في بئر بلكانة / 7، شمال العرلق

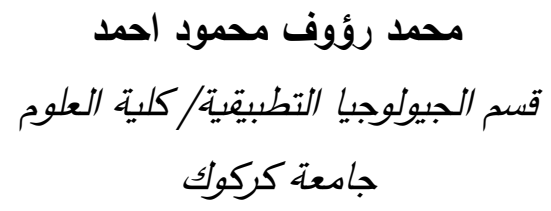

$$
\text { قبد الله سلطان شهاب الحديدي علوم الأرض/كلية العلوم }
$$$$
\text { (تاريخ الاستلام 2019/3/20 تاريخ القبول 2019/7/25) }
$$

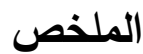

درست الفورامنيفرا الطافية لتكويني شرانش وعليجي في بئر بلكانة /7 شمال العراق بين الأعماق (1948-

$$
\text { 2240) مترًا وشخصت ستة أنطقة حياتية في التكوينين وهي من الأقدم إلى الأحدث: }
$$

1. Globotruncanita stuarti-stuartiformis Partial Range Zone.

2. Globotruncana aegyptiaca Interval Zone.

3. Gansserina gansseri Total Rang Zone.

4. Morozovella angulata Partial Range Zone .

5. Globanomalina pseudomenardii Total Rang Zone .

6. Morozovella velascoensis Partial Range Zone.

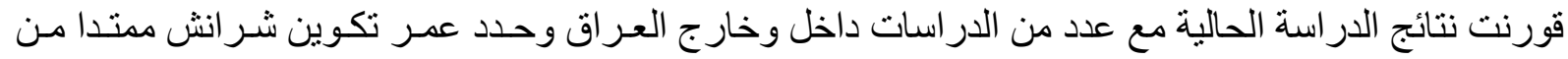

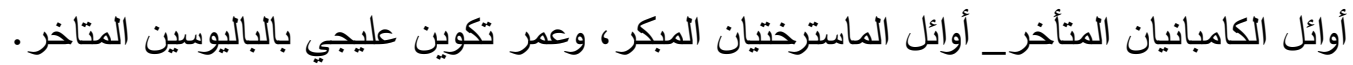

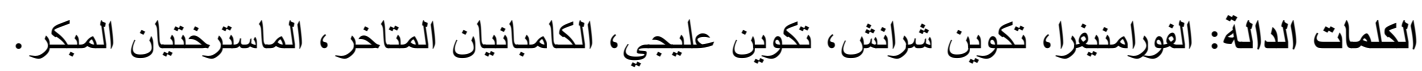

\section{Biostratigraphy Study of Planktonic Foraminifera in Shiranish Formation and its Contact with Aaliji Formation in Pulkana Well No. 7 North Iraq}

\author{
Abdullah S. S. Al-Hadidi \\ Department of Geology \\ College of Science/ Mosul University
}

\author{
Mohamed R. M. Ahmed \\ Department of Applied Geology \\ College of Science/ Kirkuk University
}

\section{ABSTRACT}

Planktonic Foraminifera is studied from Shiranish \& Aaliji formations in Pulkana Well No. 7 North Iraq between the depth intervals (1948-2240) m, six biostratigraphy zones are recognized in the formation as arranged from bottom to top:

1. Globotruncanita stuarti-stuartiformis Partial Range Zone.

2. Globotruncana aegyptiaca Interval Zone.

3. Gansserina gansseri Total Rang Zone.

4. Morozovella angulata Partial Range Zone .

5. Globanomalina pseudomenardii Total Rang Zone . 


\section{Morozovella velascoensis Partial Range Zone.}

The result of this study is compared with the work of others inside and outside Iraq, The age of the Shiranish Formation is Early Late Campanian to Earliest Maastrichtian whereas Aaliji Formation is Late Paleocene.

Keywords: Foraminifera, Shiranish Formation, Aaliji Formation, Campanian

\section{المقدمة}

يقع البئر في حقل بلكانة جنوب شرق مدينة طوز خورماتو والواقعة جنوب مدينة كركوك بحوالي (70)

كم، كما في الثكل (1) (1)

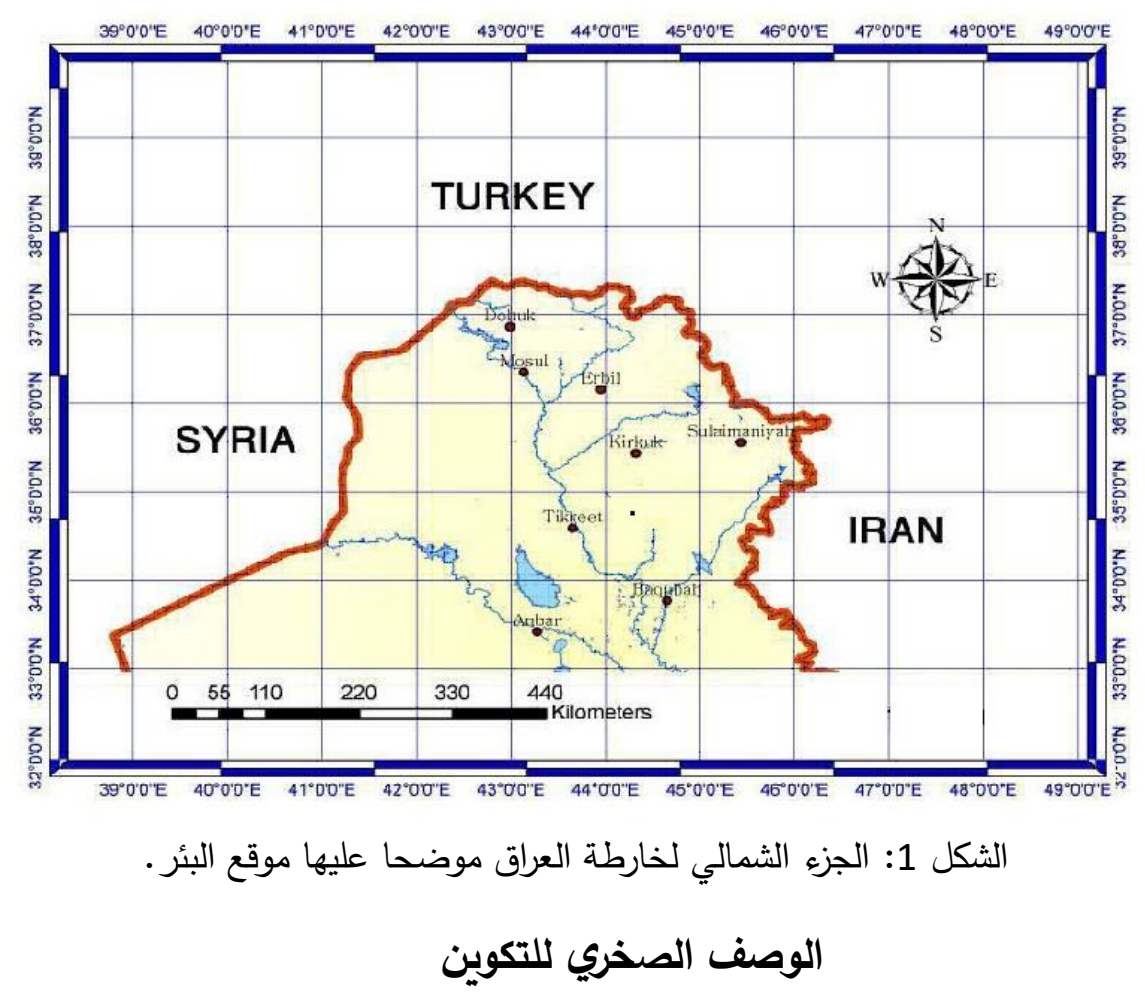

تتألف صخارية تكوين شرانش في البئر بشكل عام من صخور المارل والحجر الجيري والحجر الجيري المارلي بالتعاقب، ويتالف صخور تكوين عليجي من الحجر الجيري والحجر الجيري المارلي ، ويتميز التكوينان بكونهما غنيان بالمتحجرات الفورامنيفرا ، وتتواجد معدن الكلوكونايت بشكل حبيبات صغيرة في بعض الطبقات تكوين شرانش وهذا ان دل فانه يدل على حدوث وقفات محلية في التكوين أثناء عمليات الترسيب.

\section{الطباقية الحياتية}

اعتمادا على التوزيع الطباقي والانتثار النسبي لحشود الفورامنيفرا الطافية تم تقسيم تكوين شرانش إلى ثلاثة

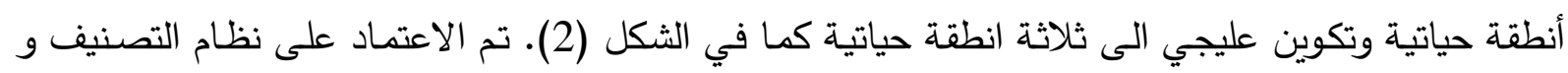

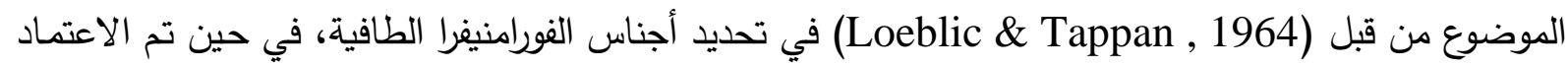

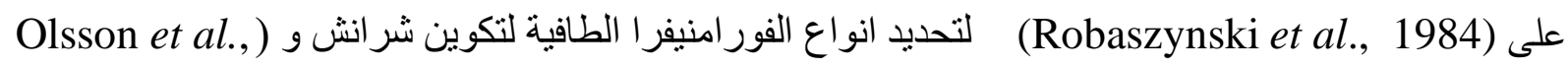
1999) في تحديد انواع الفورامنيفرا الطافية لتكوين عليجي . تم مضاهاة الأنطقة المشخصة في الدراسة الحالية 


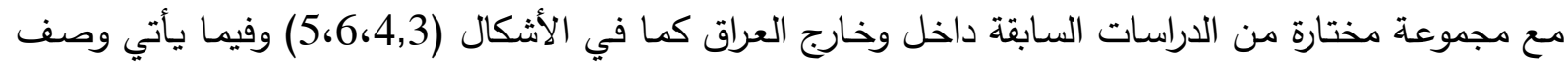
لهذه الأنطقة الحياتية من الأقدم إلى الأحدث.

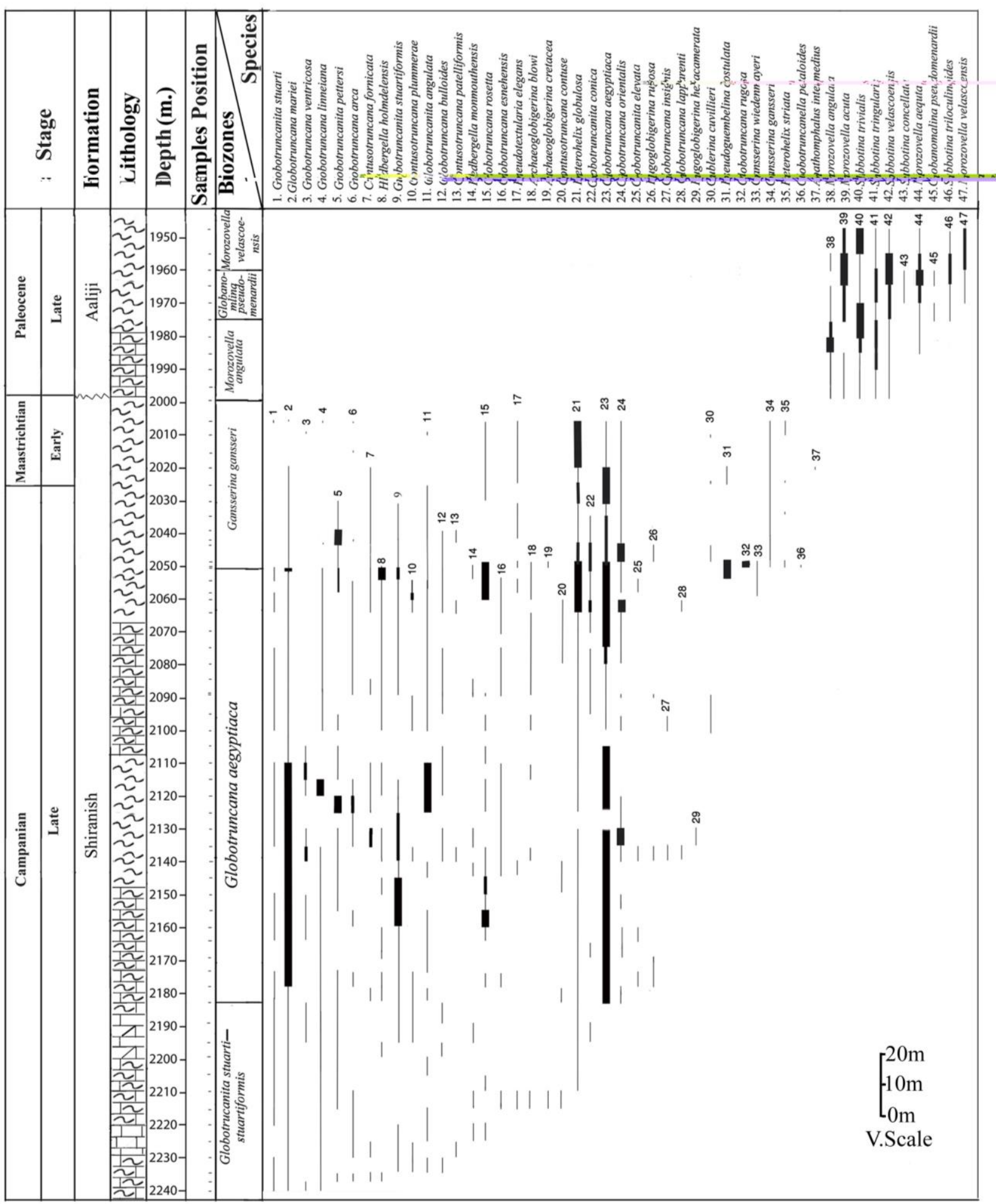

$\longrightarrow$ Rare $<(1-3) \quad \longrightarrow$ Comman $(4-5)$

الثكل 2: الددى الجيولوجي والانتشار النسبي للفورامنيفرا الطافية والانطقة الحياتية ضمن تكويني شرانش وعليجي في

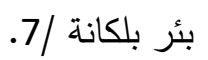




\section{1-Globotruncanita stuarti-stuartiformis Partial Range Zone}

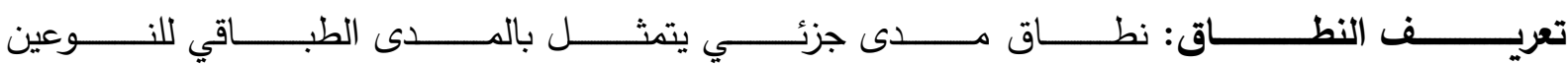

. Globotruncanita stuartiformis (Dalbiez), Globotruncanita stuarti (de Lapparent)

عمر النطاق: أواسط الكامبانيان المتأخر Middle Late Campanian.

حلود النطاق: الحد الأسفل لهذا النطاق غير ظاهر في الدراسة الحالية ويحدد عادة باختفاء النوع Globotruncanita

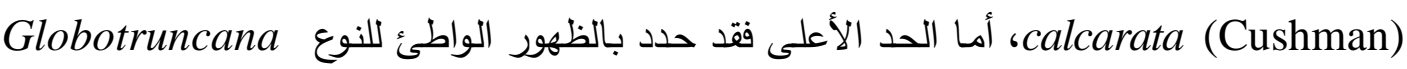
.aegyptiaca Nakkady

سمك النطاق: يبلغ سمك النطاق الحالي 56 متراً من العمق 2184 متر إلى العمق 2240 متر .

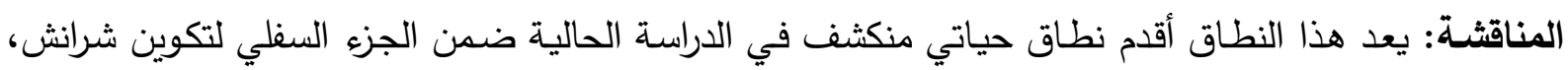

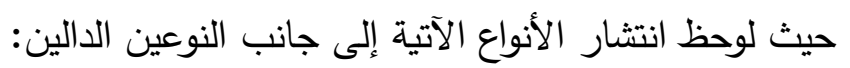

Globotruncana mariei Banner \& Blow, Globotruncana ventricosa White, Globotruncana linneiana (d'Orbigny), Globotruncanita pettersi (Gandolfi), Globotruncana arca (Cushman), Contusotruncana fornicata (Plummer), Hedbergella holmdelensis Olsson, Contusotruncana plummerae (Gandolfi), Globotruncanita angulata (Tilev), Globotruncana bulloides Vogler, Contusotruncana patelliformis (Gandolfi), Hedbergella monmouthensis (Olsson), Globotruncana rosetta (Carsey), Globotruncana esnehensis Nakkady, Pseudotextularia elegans (Rzehak), Archaeoglobigerina blowi Pessagno, Archaeoglobigerina cretacea (d'Orbigny), Contusotruncana contusa (Cushman), Heterohelix globulosa (Ehrenberg), Globotruncanita conica (White).

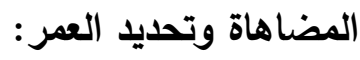

يضاهي النطاق الحالي الجزء السفلي من النطاق Lapparenti tricarinata المشخص من قبل Globotruncana بعمر الماسترختيان المبكر، كما ويضاهي مع الجزء السفلي لنطاق (Bolli, 1966) falsostuarti Globotruncanella havanensis الجزء السفلي لنطاق Globotruncanita stuarti المشخص من قبل (Maghaddam,2002) بعمر الماسترختيان المبكر ، و يضاهي نطاق Globotruncana subcarinata المحدد من قبل (Li et al., 1999)

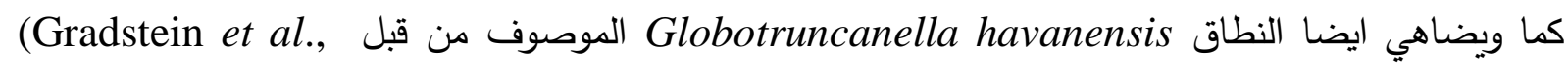
Globotruncana 2004 بعر أواسط الكامبانيان المتأخر . كما بين ان النطاق الحالي يكافئ الجزء السفلي لنطاق الناق falsostuarti

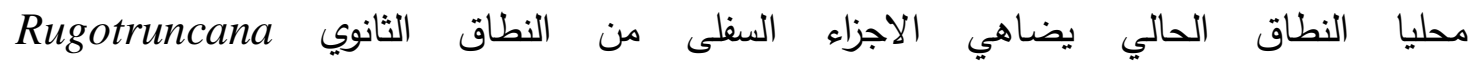
subcircumnodifer الحالي يطابق الجزء السفلي لنطاق Globotruncanella havanensis الذي حددته (Alis) (Hammoudi, 2000)، ويكافئ الجزء السفلي لنطاق Globotruncana aegyptiaca المشخص من قبل (Al-Mutwali,1996)، ويتطابق مع نطاق Globotruncanella havanensis المسجل من قبل (الناصري،2003) و ( Al-Omari 
Globotruncanita بعر الماسترختيان الاوسط، ويضاهي النطاق الحالي مع النطاق al.,2005 باق stuartiformis-stuarti الكامبانيان المتأخر ، ويتطابق مع نطاق Globotruncanita elevata-Rosita fornicata المسجل من قبل (الحديدي ، 2010) بعر اواسط الكامبانيان المتأخر .

\section{2-Globotruncana aegyptiaca Interval Zone:}

تعريف النطاق: نطاق فاصل يتمثل بالمدى الطباقي للنوع الدال Globotruncana aegyptiaca Nakkady الذي يسبق ظهور الواطئ للنوع Gansserina gansseri (Bolli). عمر النطاق: أواسط الكامبانيان المتأخر Middel Late Campanian. حدود النطاق: حدد بداية هذا النطاق بالظهور العلوي للمصنف الدال Globotruncana aegyptiaca Gansserina gansseri Nakkady

$$
\text { سمك النطاق: يبلغ سمك النطاق الحالي } 133 \text { متراً بين العمق } 2051 \text { متر إلى العمق } 2184 \text { متر . }
$$

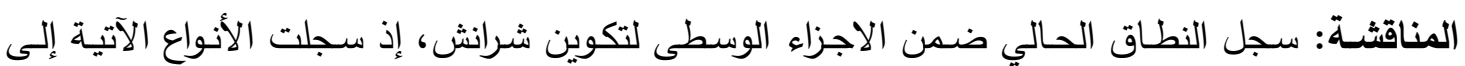

جانب الانواع السابقة المسجلة ضمن النطاق Globotruncanita stuarti- stuartiformis فضـلا

$$
\text { عن اول ظهور للنوع الدال ضمن هذا النطاق وهذه الانواع هي : }
$$

Globotruncana orientalis El Naggar, Globotruncanita elevata (Brotzen), Rugoglobigerina rugosa (Plummer), Globotruncana insignis Gandolfi, Globotruncana lapparenti (Brotzen), Rogoglobigerina hexacamerata Bronnimann, Gublerina cuvilleri kikoine, Pseudogumbelina costulata (Cushman), Globotruncana rugosa(Marie), Gansserina wiedenmayeri (Gandolfi), Htereohelix reussi(Cushman).

المضاهاة وتحديد العمر:

Lapparenti معافئ النطاق Globotruncana aegyptiaca مع الجز العلوي للنطاق tricarinata نطاق Gobaszynski et al., 1984 (لهحد من قبل Robotruncana falsostuarti بعر أواخر الماسترختيان المبكر ، ويطابق مع نطاق Globotruncana aegyptiaca المسجل من قبل (Sliter, 1989) بعمر أواخر الماسترختيان المبكر. ويكافئ هذا النطاق الجزء العلوي من نطاق Globotruncanita stuarti المحدد من قبل (Maghaddam, 2002) بعمر أواخر الماسترختيان المبكر • يكافئ النطاق الحالي مع نطاقيين Li et al., ( Rugoglobigerina hexacamerata Globotruncana aegyptiaca 1999) بعمر أواسط الكامبانيان المتأخر • ويتطابق مع نطاق Globotruncana aegyptiaca المسجل من قبل (Gradstein et al., 2004) Globotruncana falsostuarti Rugotruncana محليا النطاق المسجل يكافئ الجزء العلوي من النطاق الثانوي subcircumnodifer 
الجزء العلوي من النطاق Globotruncanella havanensis المشخص من قبل (Hammoudi, 2000) بالعمر نفسه، ويكافئ النطاق الحالي الجزء العلوي من النطاق Globotruncana aegyptiaca المسجل من قبل (Al-Mutwali,1996) بعمر الماسترختيان المبكر، ويضاهي النطاق الحالي نطاق Globotruncana aegyptiaca

الماسترختيان المبكر، ويكافئ النطاق نطاق Globotruncana aegyptiaca الذي سجله كل من - (A1) Mutwali et al., 2008 و (الحيدري، 2009) و (الحديدي، 2010) وبعمر أواخر الكامبانيان المتأخر .

\section{3-Gansserina gansseri Total Range Zone:}

تعريف النطاق: نطاق مدى كلي للنوع الدال Gansserina gansseri (Bolli). Late Campanian-Early عر النطاق: أواخر الكامبانيان المتأخر- اوائل الماسترختيان المبكر مون -Maastrichtian

حدود النطاق: شخص النطاق الحالي ضدن التتابعات العليا لتكوين شرانش التي يليها بصورة غير متوافقة طباقيا تتابعات الترشري الاسفل المتمثلة بتكوين عليجي اما حد الاسفل للنطاق فقد حدد بعد الظهور العلوي

للنطاق Globotruncana aegyptiaca.

سمك النطاق: يبلغ سمك النطاق الحالي 52 متراً اذ سجل بين الاعماق 2051 - 1999متر . المناقشة: سجل في هذا النطاق إلى جانب النوع الدال ظهور الانواع الأتية Htereohelix striata (Ehrenberg), Abathomphalus intermedius (Bolli), Globotruncanella petaloidea (Gandolifi), النطاق السابق:

Contusotruncana plummerae (Gandolfi), Globotruncana esnehensis Nakkady, Contusotruncana contusa (Cushman), Globotruncanita elevata (Brotzen), Globotruncana lapparenti (Brotzen).

المضاهاة وتحديد العمر:

يكافئ النطاق الحالي الجزء السفلي لنطاق Gansserina gansseri المسجل من قبل كل من ( Bolli, 1966)، (Robaszynski et al., 1984)، (Sliter, 1989) و (Mogaddam, 2002) بعمر الماسترختيان المتأخر • ويضاهي ايضا نطاق Gansserina gansseri و Contusotruncana contusa المحد من قبل ( Li و (et al., 1999 ويضاهي نطاق Gansserina gansseri الموصوف من قبل (Gradstein et al., 2004)، كما ويضاهي الجزء الاسفل من نطاق Gansserina gansseri المشخص من قبل (Sari, 2006) بعمر أواخر

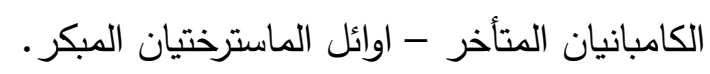
محلياً يكافئ النطاق المسجل النطاق الثانوي Globotruncana gansseri الموصوف من قبل قبل بعمر الماسترختيان المتأخر، ويضاهي النطاق الحالي ايضا الجزء الاسفل من نطاق (Abawi et al., 1982) Gansserina gansseri (الناصري ،Al-Omari et al., 2005) الذي يمثل عمر الماسترختيان المتأخر • ويتطابق النطاق الحالي مع نطاق Gansserina gansseri المسجل من قبل (الحيدري، 2009) بعمر اواخر الكامبانيان 
المتاخر ، ويتطابق مع نطاق Gansserina gansseri المشخص من قبل (الحديدي، 2010) بعر ممتد من أواخر الكامبانيان المتأخر إلى اوائل الماسترختيان المبكر وبذلك يكون عمر النطاق الحالي أواخر الكامبانيان المتاخر_أوائل الماسترختيان المبكر .

\section{4-Morozovella angulata Partial Range Zone}

Morozovella angulata (White) تعريف النطاق: نطاق مدى جزئي يحدد بين اول ظهور للنوع الداليين Globanomalina pseudomenardii (Bolli)

Early Late Paleocene عمر النطاق: اوائل الباليوسين المتأخر سمك النطاق: يبلغ سمك هذا النطاق 24 متراً من العمق 1999متر إلى 1975 متر .

حدود النطاق: الحد الأسفل للنطاق يمثل اختفاء الفورامنيفرا الطافية على الكريتاسي الاعلى بينما يمثل ظهور الواطئ النوع الدال Golli) Gobanomalina pseudomenardii (Bolli الحد الأعلى للنطاق. المناقشة: تظهر حشود الفورامنيفرا بشكل وفير في بعض النماذج في حين تظهر بشكل قليل في نماذج اخرى ، إذ تم تسجيل الانواع الأتية إلى جانب النوع الدال

Morozovella acuta (Toulmin), Subbotina trivialis(Subbotina), Subbotina tringularis (White), Subbotina velascoensis (Cushman) , Subbotina concellata(Blow), Morozovella aequta(Cushman \& Renz).

المضاهاة وتحديد العمر:

Globorotalia angulata معافئ النطاق الحالي Morozovella angulata مع نطاق المشخص من قبل (Postoma,1971) ، ويضاهي ايضا النطاقين Globorotalia pusilla pusilla و Morozovella المسجلين من قبل (Stainforth et al.,1975) ويطابق نطاقي Globorotalia angulata Morozovella angulata و pusilla pusilla الباليوسين الاوسط ، ويطابق النطاق الحالي ايضا النطاقين Igorina albeari و Morozovella angulata المشخصيين من قبل (Olsson et al., 1999) ويضاهي نطاقي Igorina pusilla pusilla و Morozovella angulata اوائل الباليوسين المتأخر.

داخل العراق يضاهي النطاق الحالي نطاقي Globorotalia pusilla pusilla و Globorotalia

angulata الموصوف من قبل (المتولي، 1983) و (Al-Hashimi \& Amer, 1985) و (غفور، 1988)، ويطابق مع نطاق Morozovella angulata المسجل من قبل (الحديدي، 2008a) بعمر اواخر الباليوسين الاوسط، ويضاهي نطاق المسجل من قبل (الوزان، 2007) و(المتولي والوزان، 2010) بعدر اوائل الباليوسين المتأخر وبذلك يكون عمر النطاق اوائل الباليوسين المتأخر . 
تعريف النطاق: نطاق مدى كلي للمصنف الدال (Bolli ) Globanomalina pseudomenardii ) عiddle Late Paleocene عمر النطاق: أواسط الباليوسين المتأخرى

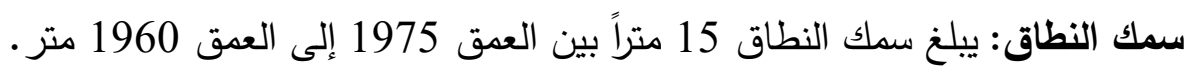

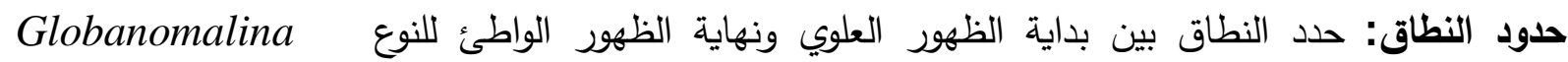
. pseudomenardii(Bolli)

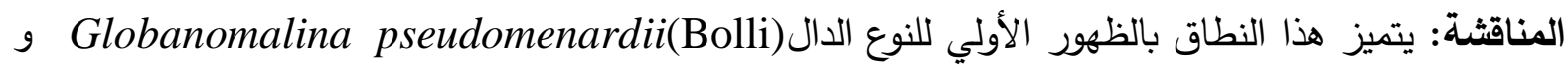
Subbotina triloculinoides (Plummer)

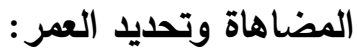

يكافئ النطاق الحالي نطاق Globorotalia pseudomenardii pseudomenardii Planorotalia ويطابق نطاق (Postoma,1971) و (Stainforth et al.,1975) الموصوف من قبل (Toumarkine \& Luterbacher, 1985)و (1996, Arenilas Moline) بعر اوائل Olsson ( الباليوسين المتاخر ، ويضاهي نطاق Globanomalina pseudomenardii المشخص من قبل (El-Nady \& Shahin,2001) و (et al., 1999 ) (El-Nady,2005) ضمن الجزء الاوسط من الباليوسين المتأخر. محلياً يضاهي النطاق الحالي الجزء الاسفل من نطاق Globorotalia velascoensis الموصوف من

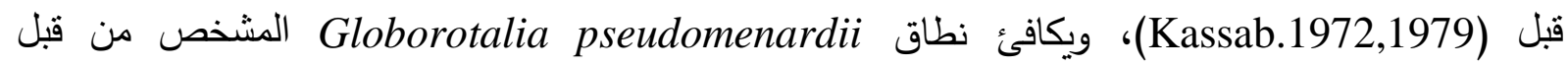
(المتولي، 1983) و (Al-Hashimi \& Amer, 1985) و (غفور، 1988)، كما ويطابق مع نطاق Globanomalina pseudomenardii الباليوسين المتأخر ، ويضاهي ايضا نطاق Globanomalina pseudomenardii المسجل من قبل (الوزان، 2007) و (المتولي والوزان، 2010) بعمر اواسط الباليوسين المتاخر • وبذلك يكون عمر النطاق الحالي اواسط

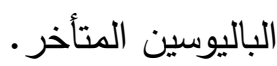

\section{6- Morozovella velascoensis Partial Range Zone}

تعريف النطاق :نطاق مدى جزئي للنوع الدال Morozovella velascoensis(Cushman)

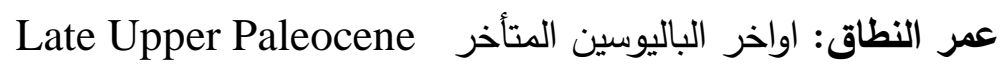
حدود النطاق: حدد الحد الاسفل للنطاق بعد الظهور العلوي للنوع Globanomalina pseudomenardii

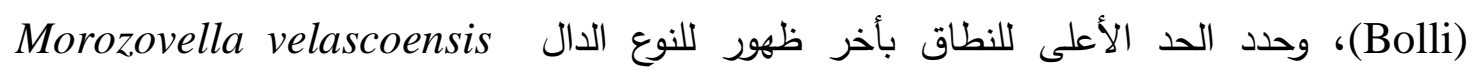

.(Cushman)

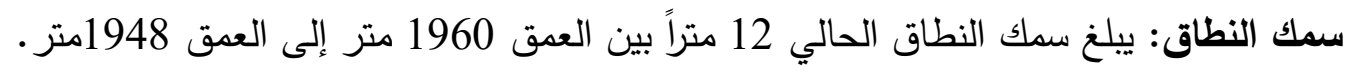
المناقثة: يتميز هذا النطاق بوفرة لبعض الانواع المسجلة وحفظها الجيد فقد سجل ضمن النقال النطاق الحالي استمرار الانواع المشخصة السابقة. 
يضاهي النطاق الحالي نطاق Globorotalia velascoensis المحدد من قبل (Postoma,1971) و (Stainforth et al.,1975)، كما يكافئ ايضا نطاق Morozovella velascoensis المشخص من قبل Lgarim \& Morozovella velascoensis (Toumarkine \& Luterbacher, 1985) lacvigata ايضا نطاق Morozovella velascoensis الموصوف من قبل كل من (Olsson et al., 1999 ) و)-El (Nady \& Shahin , 2001 محليا يكافئ هذا النطاق الجزء الاعلى من نطاق Globorotalia velascoensis المشخص من قبل (Kassab, 1972, 1979) (المتولي، 1983) و (Al-Hashimi \& Amer, 1985) و (غفور، 1988) بعمر اواخر الباليوسين المتأخر ، ويكافئ النطاق Morozovella velascoensis المسجل من قبل (الحديدي، 2008a، 2008b) ضمن العمر نفسه، ويضاهي نطاق Morozovella aequa /Acarinia esnaensis الموصوف من قبل (الوزان، 2007)، (المتولي و الوزان، 2010) بعمر اواخر الباليوسين المتأخر • يظهر مما تقدم في أعلاه بان عمر النطاق الحالي أواخر الباليوسين المتأخر. 


\begin{tabular}{|c|c|c|c|c|c|c|c|c|c|c|c|}
\hline $\begin{array}{c}\text { Late } \\
\text { Campanian }\end{array}$ & \multicolumn{10}{|c|}{ Maastrichtian } & \multirow{2}{*}{ 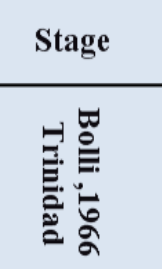 } \\
\hline 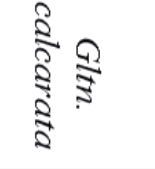 & \multicolumn{3}{|c|}{ 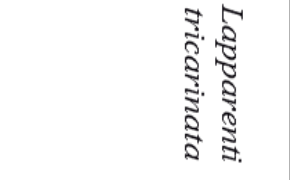 } & \multicolumn{3}{|c|}{ 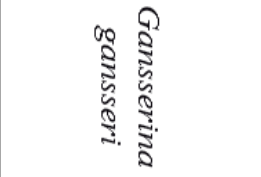 } & \multicolumn{4}{|c|}{ 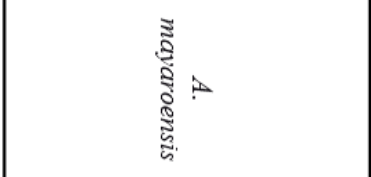 } & \\
\hline$\frac{5}{\frac{5}{3}} \frac{7}{5}$ & \multicolumn{3}{|c|}{ 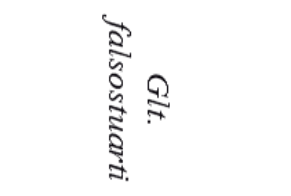 } & \multicolumn{3}{|c|}{ 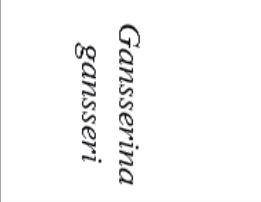 } & \multicolumn{4}{|c|}{ 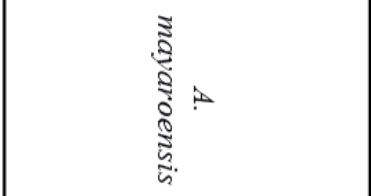 } & 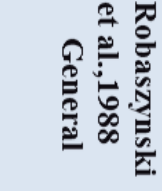 \\
\hline 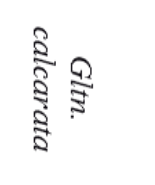 & 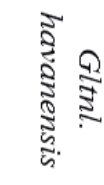 & \multicolumn{2}{|c|}{ 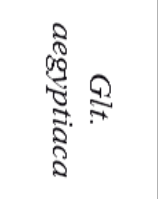 } & \multicolumn{3}{|c|}{ 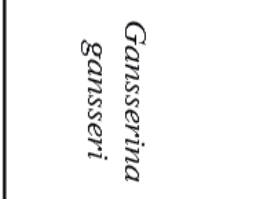 } & \multicolumn{4}{|c|}{ 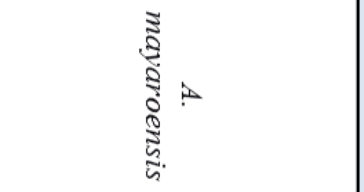 } & 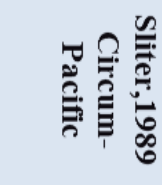 \\
\hline 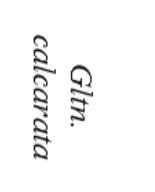 & \multicolumn{3}{|c|}{ 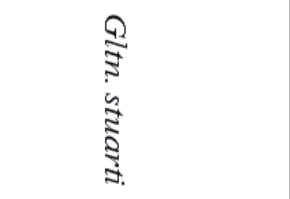 } & \multicolumn{3}{|c|}{ 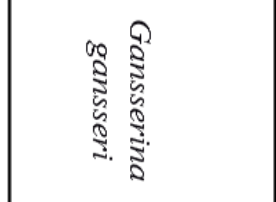 } & \multicolumn{4}{|c|}{ 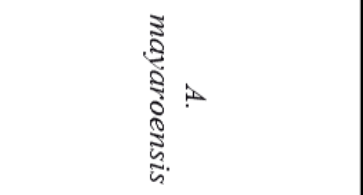 } & 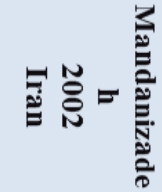 \\
\hline \multicolumn{5}{|c|}{ Late Campanian } & \multicolumn{2}{|l|}{ Early } & \multicolumn{4}{|c|}{ Late } & Stage \\
\hline $\begin{array}{c}\frac{5}{0} \\
\vdots \\
0 \\
0 \\
\frac{1}{2} \\
0 \\
0\end{array}$ & 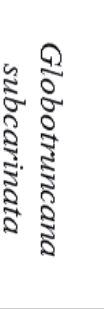 & 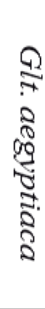 & 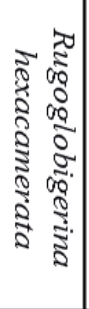 & 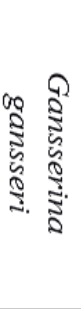 & 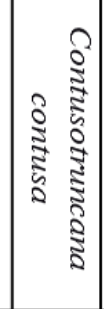 & 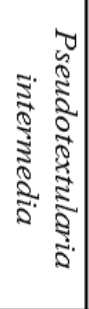 & 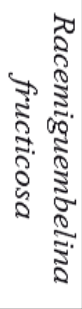 & 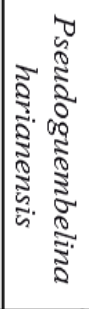 & 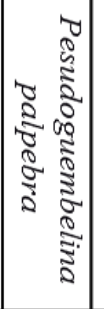 & 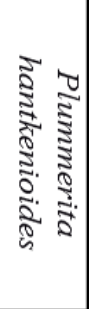 & 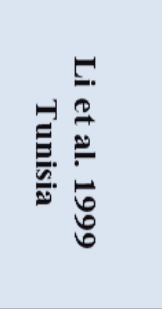 \\
\hline$\frac{5}{3}$ & 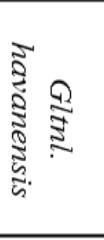 & & 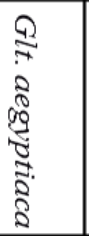 & 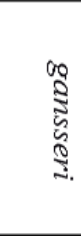 & 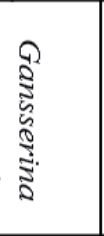 & & & 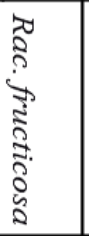 & 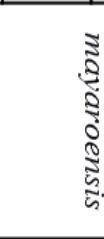 & + & 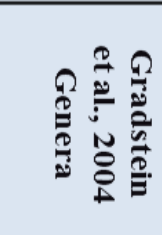 \\
\hline $\begin{array}{l}5 \\
0 \\
0 \\
0 \\
0 \\
0 \\
0 \\
0 \\
0\end{array}$ & 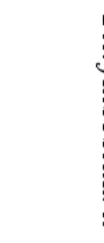 & & & & 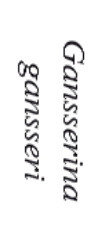 & & & 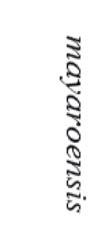 & s & & 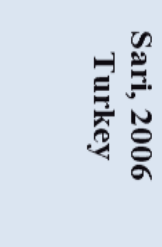 \\
\hline & 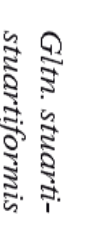 & & 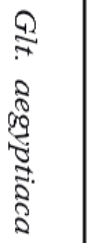 & 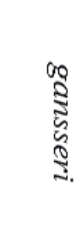 & 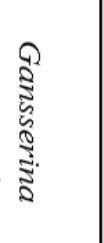 & & & & & & 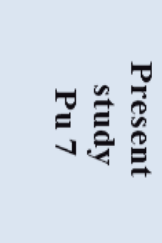 \\
\hline
\end{tabular}


دراسة الطباقية الحياتية للفورامنيفرا الطافية لتكوين شرانش وطبيعة تماسه مع تكوين عليجي .

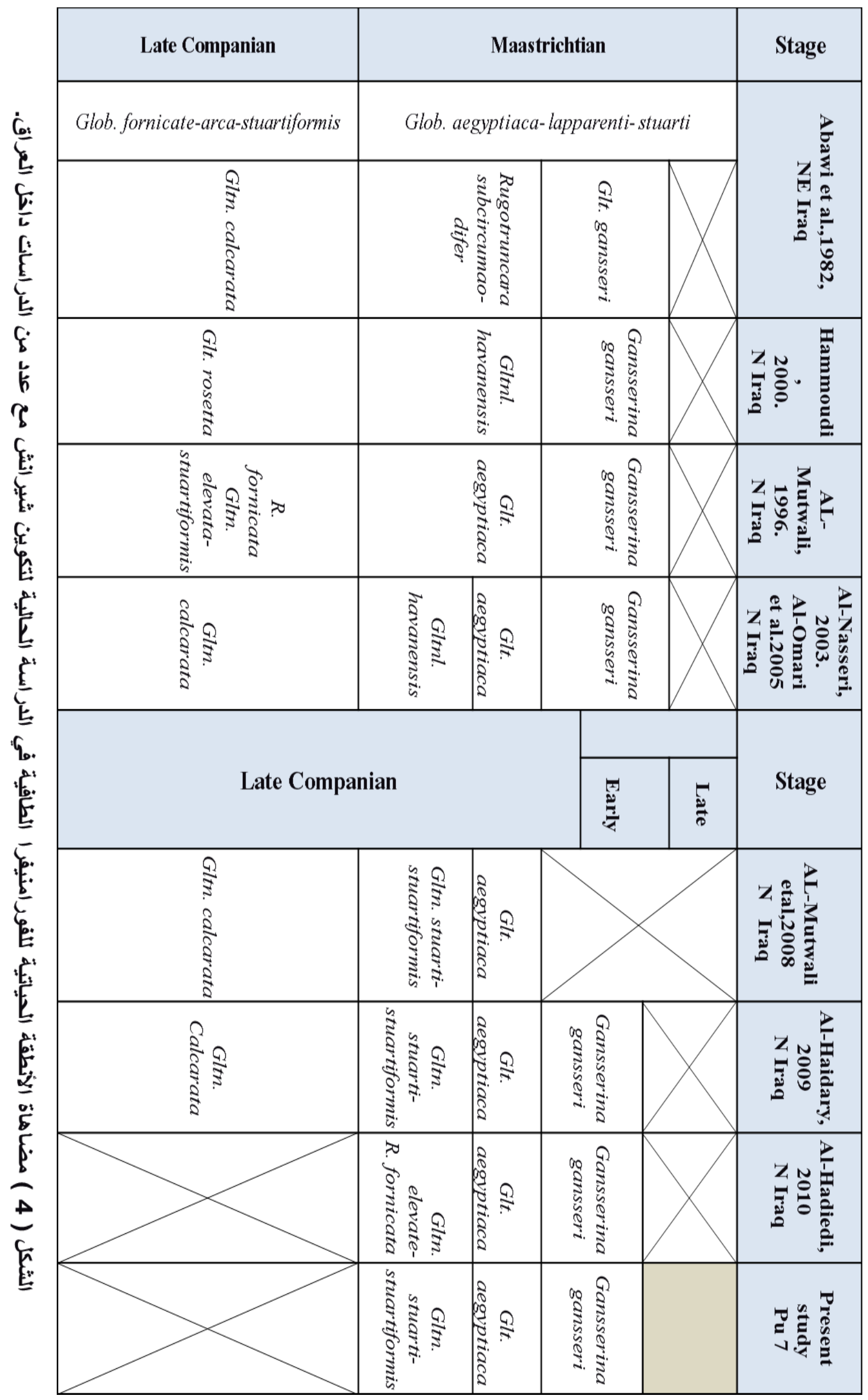




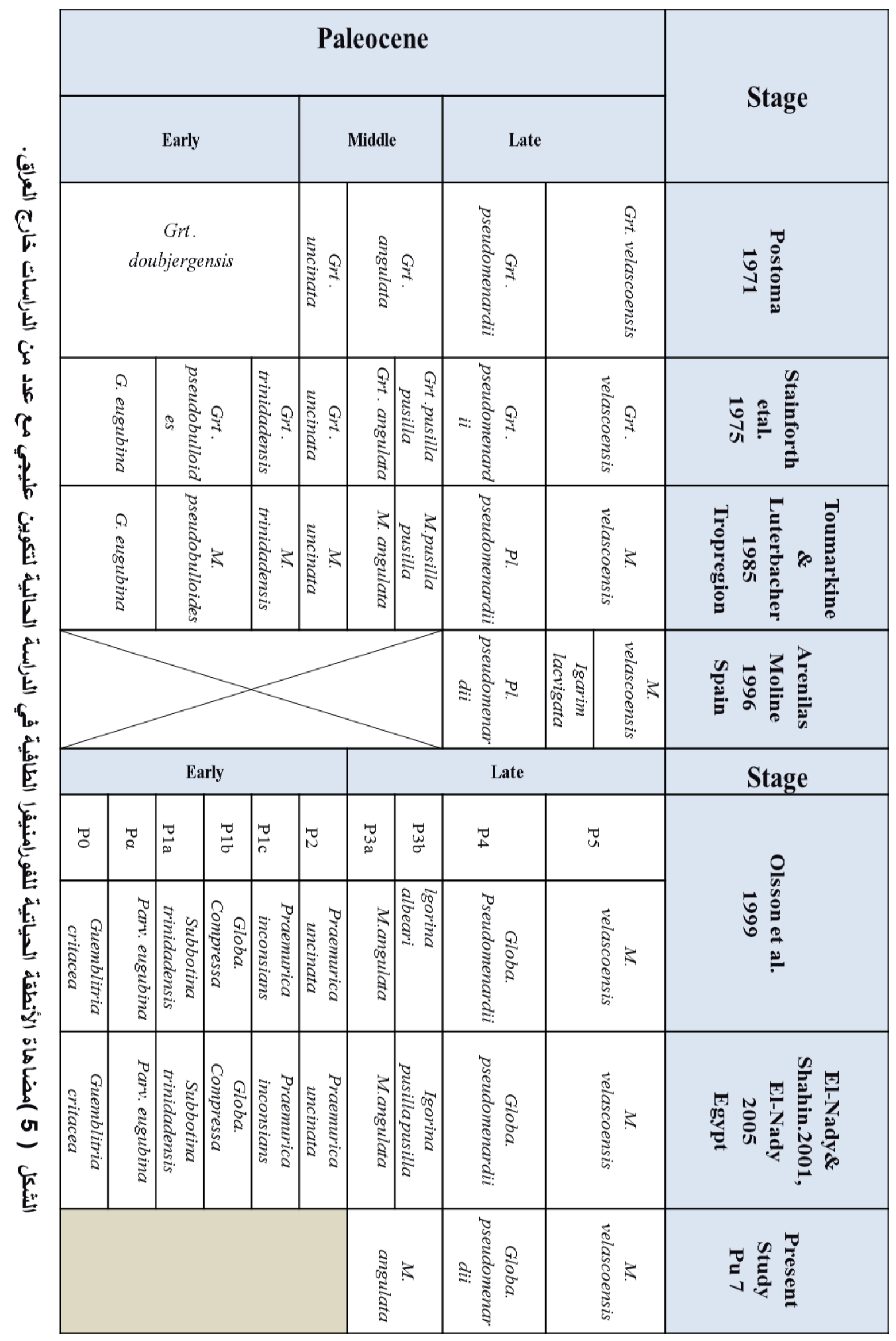




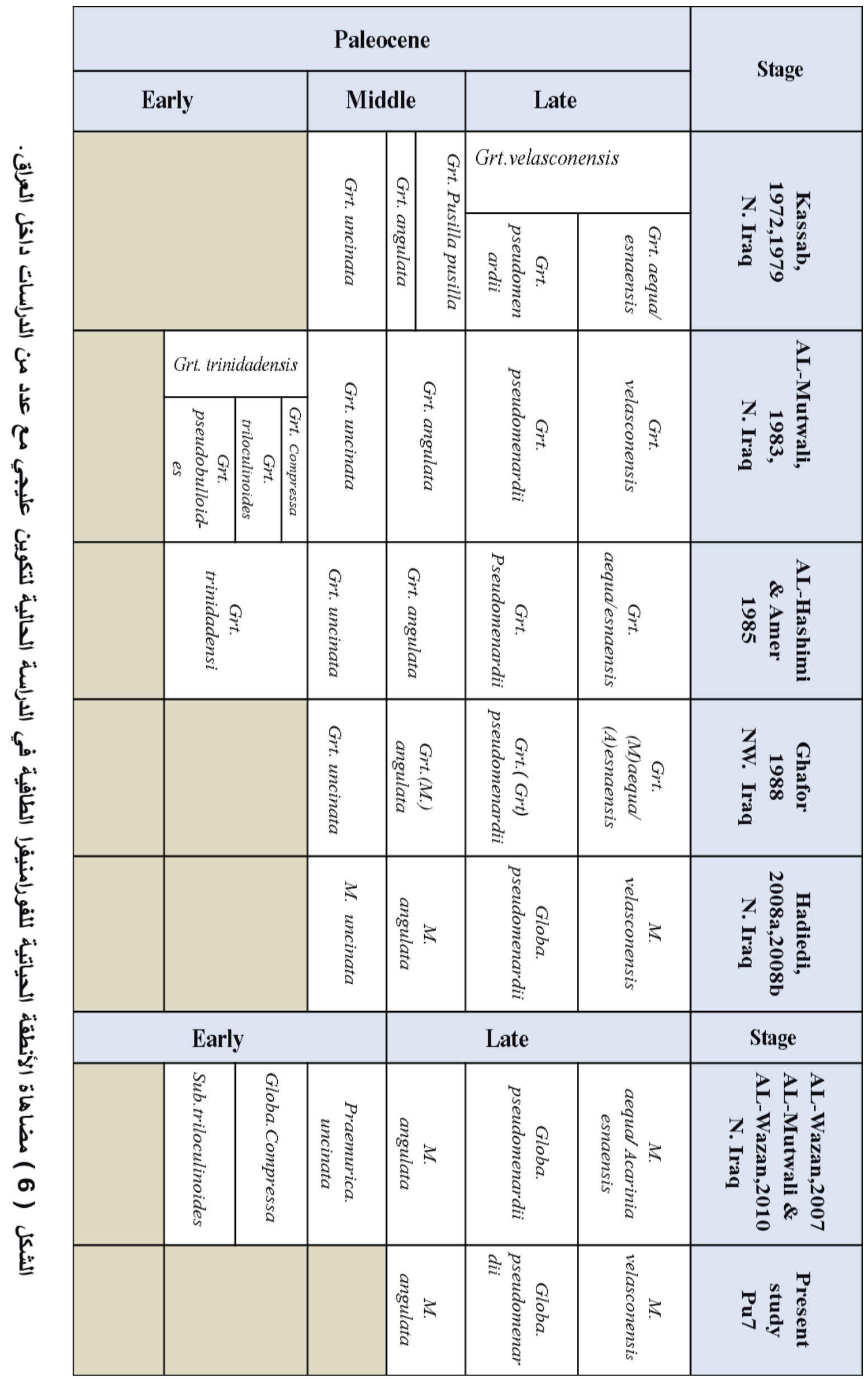




\section{الاستنتاجات}

1. تم تشخيص (37) نوعاً من أنواع الفورامنيفرا الطافية تعود الى (13) جنساً ضمن تكوين شرانش و (10) أنواع من الفورامنيفرا الطافية تعود الى (3) اجناس ضمن تكوين عليجي. 2. حد التماس العلوي لتكوين شرانش غير متوافق طباقيا مع تكوين عليجي بدلالة الانقطاعات الاحيائية لحشود الفورامنيفرا الطافية عبر الحد الفاصل بين عصر الكريتاسي الاعلى وعصر الترشري الأسف.

3. بينت الدراسة ايضا ان الحد التماس بين تكوين شرانش وعليجي في بئر بلكانة / 7 عند العمق 1999 متراً بعدما كانت مثبتة عند العمق 2050 متراً في التقرير النهائي لشركة نفط الثمال وبذلك يكون سمك تكوين شرانش 241 متراً.

4. تم تشخيص ستة أنطقه حياتية ضمن تكويني شرانش وعليجي استنادا الى التوزيع الطباقي للفورامنيفرا الطافية وهي كالآتي من الأقدم إلى الأحدث:

1. Globotruncanita stuarti-stuartiformis Partial Range Zone.

2. Globotruncana aegyptiaca Interval Zone.

3. Gansserina gansseri Total Rang Zone.

4. Morozovella angulata Partial Range Zone .

5. Globanomalina pseudomenardii Total Rang Zone .

6. Morozovella velascoensis Partial Range Zone.

5. حدد عمر تكوين شرانش ضمن المقطع قيد الدرس بعد مضاهاة الأنطقة الحياتية المسجلة مع دراسات داخل وخارج القطر بعمر أوائل الكامبانيان المتأخر_ أوائل الماسترختيان المبكر. 6. حدد عمر تكوين عليجي في الدراسة الحالية بعمر الباليوسين المتأخر.

\section{المصادر العربية}

الناصري، عمر عادل محمد يعقوب، 2003: طباقية التتابع لدورة الكامبانيان الاعلى - الماسترختيان في ابار مختارة من حقول صفية، عين زالة، بطمة وجبل قند شمال غرب العراق. رسالة ماجستير غير منشورة، كلية العلوم، جامعة الموصل، 88 صفحة.

العمري، فاروق صنع الله، وحداد، صباح نوري، والناصري، عمر عادل محمد، 2005: الطباقية الحياتية للفورامنيفرا الطافية لتكوين شرانش (الكامباني المتاخر - الماسترختي الاوسط) في بئر بطمة (15)، شمال غرب العراق. المجلة العراقية لعلوم الارض، المجلد 5، العدد 1، الصفحات 32-43. 
الحيدري، ليلى يوسف فرمان، 2009: طباقية وبيئة ترسيب تكوين بخمة وطبيعة تماسه مع تكوين شرانش في منطقة دهوك. رسالة ماجستير غير منشورة، كلية العلوم، جامعة الموصل، 131 صفحة.

الحديدي، عبدالله سلطان شهاب، 2010: الطباقية الحياتية للفورامنيفرا الطافية لتكوين شرانش في بئر مخمور (1) منطقة مخمور شمال العراق. المجلة العراقية لعلوم الارض، المجلد 10، العدد 2، الصفحات 2 - 19.

\section{المصادر الاجنبية}

Loeblich, A.R. \& Tappan, H., 1964: Sarcodina chiefly Thecamoebians and foraminifera. In: Moor, R.C. (ed.) Treatise on Invertebrate Paleontology, Protista 2, pt. Univ. Kansas Press, 900p.

Robaszynski, F.; Caron, M.; Gonzalez, J. M. and Wonders, A. A. H., 1984: Atlas of Late Cretaceous Globotruncananids. Revue de Microp., v.26, n.3,4, pp.145305.

Olsson, R. K., Hemleben, C.,Berggren ,W . A. and Huber, B.T, 1999: Atlas of Paleocene Planktonic Foraminifera, Smithsonian contributions to paleo bio. $252 \mathrm{p}$.

Bolli, H. M., 1966: Zonation of Cretaceous to Pliocene marine sediments based on planktonic foraminifera. Bol. Inform. Assoc. Venezuelana Geo. Min. Pet. In a(1), pp.3-32.

Sliter, W.V., 1989: Biostratigraphic zonation for Cretaceous planktonic foraminifers examined in thin section. Jou. Foram. Res., v. 19, n. 1, pp. 1-19.

Mogaddam, H.V., 2002: Biostratigraphic study of the Ilam and Gurpi Formation based on planktonic foraminifera in SE of Shiraz, Iran, Jou. of Sci. Iran, v. 13, n. 4, pp. $339-356$.

Li, L., Keller, G. and Stinnesbeck, W.,1999: The Late Campanian and Maastrichtian in Northwestern Tunisia: Palaeoenvironmental inferences from lithology, macrofauna and benthic foraminifera. Cret. Res. v. 20, pp. 231-252.

Gradstein, F.M., Ogg, J.G. \& Smith, A.G. (Eds.), 2004: Geological Time Scale 2004. Cambridge Univ. Press, 500p.

Sari, B., 2006: Upper Cretaceous planktonic foraminiferal biostratigraphy of the Bey Daglari Autochthon in the Korkuteli area, Western Taurides, Turkey. Jou. Foram. Res., v. 36, n. 3, pp. 241-261.

Abawi, T. S.; Abdel-Kireem, M. R. and Yousef, G. M., 1982: Planktonic foraminiferal stratigraphy of Shiranish Formation, Sulaimaniah- Dohok region Northeastern Iraq. Revista Espnola de Microp. v. 14, n. 1, pp.153-164.

Hammoudi, R. A., 2000: Planktonic foraminiferal biostratigraphy of the Shiranish Formation (Upper Cretaceous) in Jambur well No.13, Northern Iraq. Raf. Jour. Sci., 11(4), pp.50-58. 
Al-Mutwali, M. M., 1996: Planktonic foraminiferal biostratigraphy of the Shiranish Formation, Khashab well no.1, Hemren area, North eastern Iraq. Jou. Geo. Sci. Iraq. v. 7, n. 1, pp.129-136.

Al-Mutwali, M.M., Al-Bana, N.Y. \& Al-Ghrear, J.S., 2008: Microfacies and sequences stratigraphy of the late Campanian Bekhme Formation in Dohuk area, North Iraq. GeoArabia, v. 13, n.1, pp.39-54.

Postuma, J. A., 1971: Manual of planktonic foraminifera. Elsevier Publ. Co., Amsterdam, $420 \mathrm{p}$.

Stainfort, R. M., Lamp, J.L., Luterbacher, H., Beard, J. H. \& Jeffords, R.M., 1975: Cenozoic Planktonic Foraminifera zonation \& Characterstics of index forms. Univ. Kansas Pale. Contr. Articale, pp.62-425.

Toumarkine, M. and Luterbacher, H.P., 1985: Paleocene \& Eocene Planktonic foraminifera .In H. M. Bolli, J.B. Sauuders, and K. perch -Nelsen, editor, Planktonic stratigraphy, Cambridge Univ. Press. pp.87-154.

Molina, E., Arenillas, I. \& Gonazlvo, C., 1996: Filed trip guide to the Paleocene Middle Eocene of Agost, Caravca \& Almaedilla section, Actos Early Paleogene Stage Boundaries, Zaragoza, pp.73-103.

El-Nady, H. I. A. \& Shahin, A.M.,2001: Planktonic foraminiferal biostratigraphy \& Paleobathymetry of the Late Cretaceous-Early Tertiory succession at Northeast Sinai, Egypt. Egypt, Jour. Paleon. v.1, pp.193-227.

El-Nady, H. I. A., 2005: The impact of Paleocene / Eocene (P/E) boundary events in northern Sinai, Egypt:Planktonic foraminiferal biostratigraphy and faunal turnovers, Paleobio. Geneve ,v.24, n.1, pp.1-16 


\section{PLATE 1}

Fig. 1a: Globotruncanita stuarti (de Lapparent). Spiral side.

Shiranish Formation Sample No. 57 x100.Pu.7.

Fig. 1b: Globotruncanita stuarti (de Lapparent). Spiral side.

Shiranish Formation Sample No. 57 x100.Pu.7.

Fig. 2a: Globotruncanita stuartiformis (Dalbiez). Spiral side.

Shiranish Formation Sample No. 61 x100.Pu.7.

Fig. 2b: Globotruncanita stuartiformis (Dalbiez). Side view.

Shiranish Formation Sample No. 61 x100.Pu.7.

Fig. 3a: Globotruncanella petaloidea (Gandolfi). Spiral side.

Shiranish Formation Sample No. 22 x100.Pu.7.

Fig. 3b: Globotruncanella petaloidea (Gandolfi). Umbilical side.

Shiranish Formation Sample No. 22 x100.Pu.7.

Fig. 4: Globotruncanita conica (White). Spiral side.

Shiranish Formation Sample No. 20 x100.Pu.7.

Fig. 5: Heterohelix globulosa (Ehrenberg). Side view.

Shiranish Formation Sample No. 20 x100. Pu.

Fig. 6a: Globotruncana bulloides Vogler. Spiral side.

Shiranish Formation Sample No. 37x100.Pu.7.

Fig. 6b: Globotruncana bulloides Vogler. Side view.

Shiranish Formation Sample No. 37x100.Pu.7.

Fig. 7a: Globotruncana esnehenses Nakkady. Spiral side.

Shiranish Formation Sample No.27x100. Pu.7.

Fig. 7b: Globotruncana esnehenses Nakkady. Umbilical side.

Shiranish Formation Sample No. 27x100. Pu.7.

\section{PLATE 2}

Fig. 1a: Globotruncana aegyptiaca Nakkady. Spiral side.

Shiranish Formation Sample No. 37 x100. Pu.7.

Fig. 1b: Globotruncana aegyptiaca Nakkady. Umbilical side.

Shiranish Formation Sample No. 17x100. Pu.7.

Fig. 2: Gansserina gansseri (Bolli). Side view.

Shiranish Formation Sample No. 21 x100. Pu.7.

Fig. 3: Morozovella angulata (White). Spiral side.

Aaliji Formation Sample No. 10 x100. Pu.7.

Fig. 4: Morozovella velascoensis (Cushman). Spiral side.

Aaliji Formation Sample No. 2 x100. Pu.7.

Fig. 5: Globanomalina pseudomenardii (Bolli). Spiral side. Aaliji Formation Sample No. 5 x100. Pu.7.

Fig. 6: Morozovella acuta (Toulmin). Spiral side.

Aaliji Formation Sample No. 6 x100. Pu.7.

Fig. 7: Morozovella aequa (Cushman \& Renz). Spiral side.

Aaliji Formation Sample No. 8 x100. Pu.7.

Fig. 8: Subbotina triangularia (White). Spiral side.

Aaliji Formation Sample No. 4 x100. Pu.7.

Fig. 9: Subbotina velascoensis (Cushman). Spiral side.

Aaliji Formation Sample No. 8 x100. Pu.7.

Fig. 10: Subbotina cancellata Blow. Spiral side.

Aaliji Formation Sample No. 4 x100. Pu.7. 


$$
\begin{gathered}
\text { عبدالله سلطان شهاب الحديدي و محمد رؤوف محمود احمد } \\
\text { PLATE } 1
\end{gathered}
$$

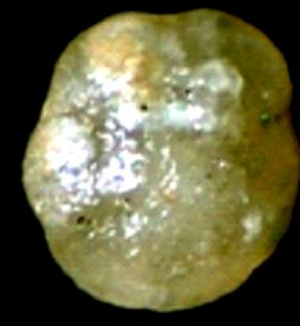

Fig. 1a

Fig. 2b

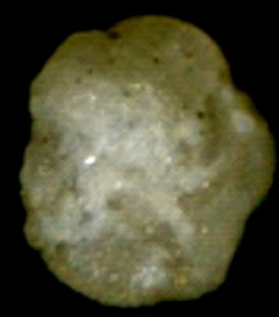

Fig. 4

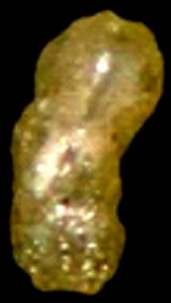

Fig. 6b

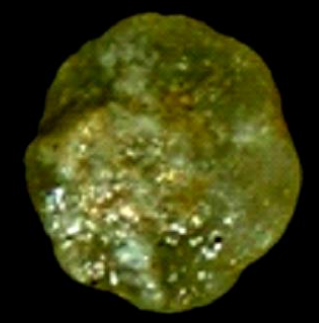

Fig.1b

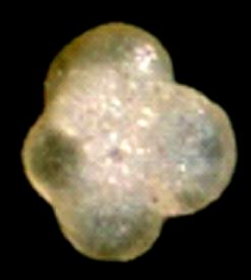

Fig. $3 a$

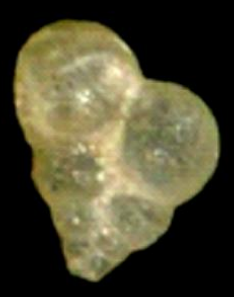

Fig. 5

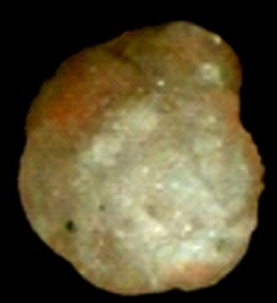

Fig. $7 a$

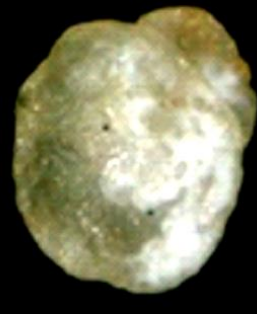

Fig. $2 a$

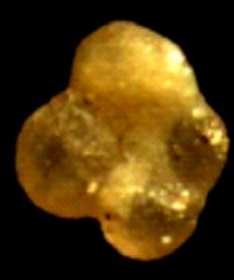

Fig. 3b

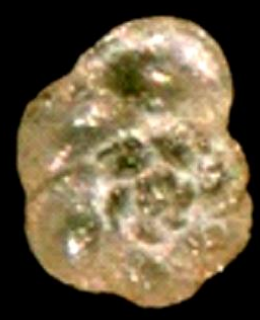

Fig. $6 a$

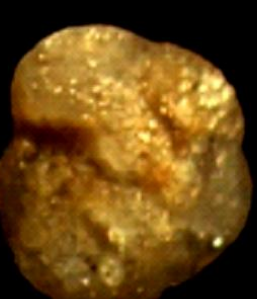

Fig. 7b 


\section{PLATE 2}

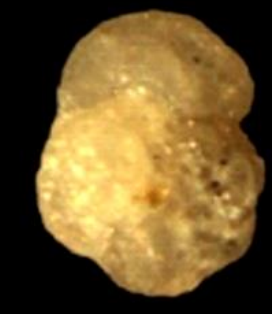

Fig. 1a

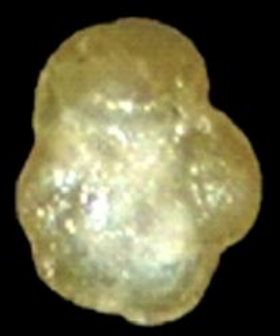

Fig.1b

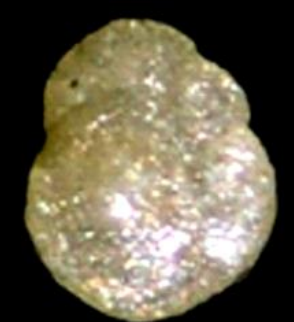

Fig. 4

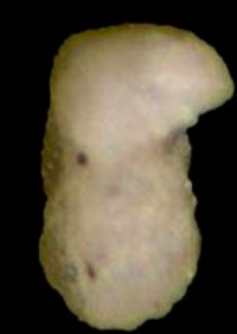

Fig. 2
Fig. 3

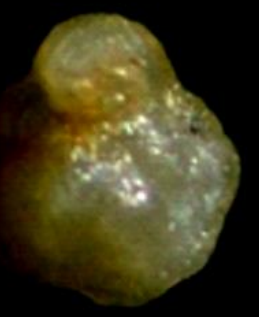

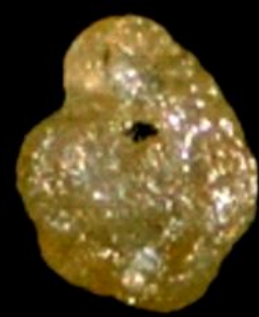

Fig. 6

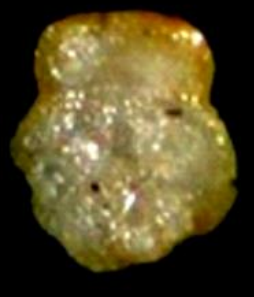

Fig. 7

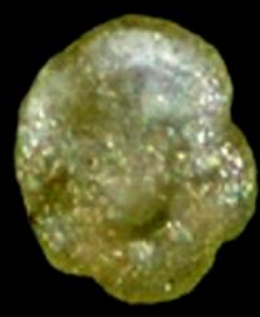

Fig. 5
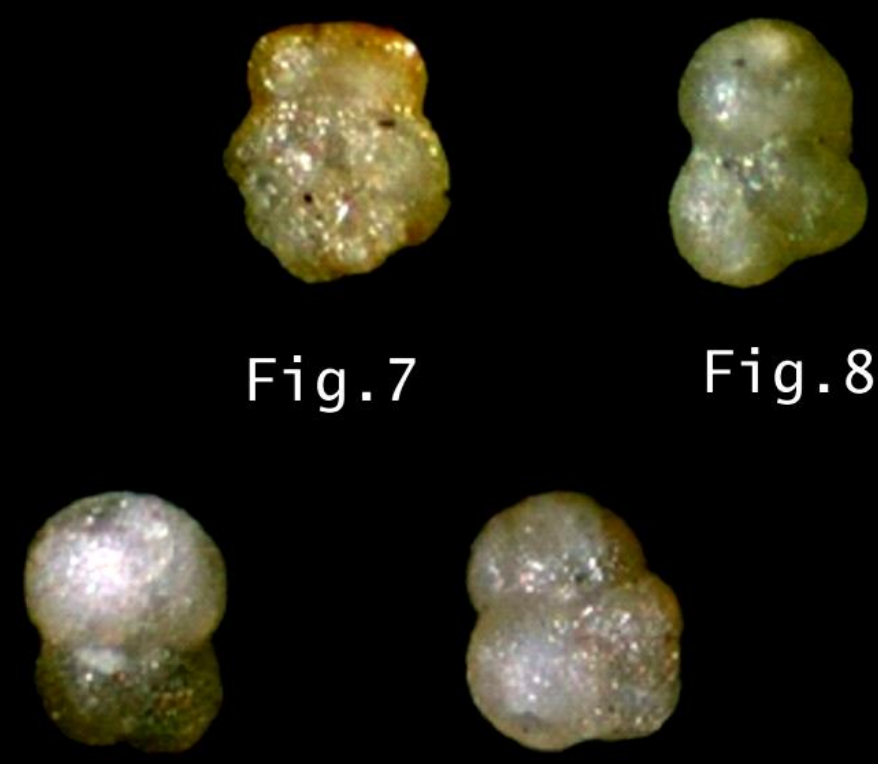

Fig. 8

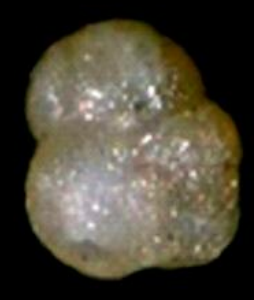

Fig. 10 